\title{
Phenotypic and genotypic heterogeneity in the Lynch syndrome: diagnostic, surveillance and management implications
}

\author{
Henry T Lynch*,1, C Richard Boland ${ }^{2}$, Gordon Gong ${ }^{1}$, Trudy G Shaw ${ }^{1}$, \\ Patrick M Lynch ${ }^{3}$, Riccardo Fodde ${ }^{4}, J^{2}$. F Lynch ${ }^{1}$ and Albert de la Chapelle ${ }^{5}$
}

\author{
${ }^{1}$ Department of Preventive Medicine, Creighton University School of Medicine, Omaha, NE, USA; ${ }^{2}$ Division of \\ Gastroenterology, Baylor University Medical Center, Dallas, TX, USA; ${ }^{3}$ Division of GI Oncology, MD Anderson Cancer \\ Center, Houston, TX, USA; ${ }^{4}$ Department of Pathology, Josephine Nefkens Institute, Erasmus University Medical Center, \\ Rotterdam, The Netherlands; ${ }^{5}$ Human Cancer Genetics Program, Comprehensive Cancer Center, The Ohio State \\ University, Columbus, OH, USA
}

Lynch syndrome is the most common form of hereditary colorectal cancer (CRC). This review covers the cardinal features of Lynch syndrome with particular emphasis upon its diagnostic criteria, molecular genetics, natural history, genetic counseling, surveillance and management. Considerable attention has been given to the etiologic role of mismatch repair (MMR) genes as well as low penetrance alleles and modifier genes. The American founder mutation, a deletion of exons 1-6 of $M S H 2$, is discussed in some detail, owing to its high frequency in the US (19000-30000 carriers). Genetic counseling is essential prior to patients' undergoing DNA testing and again when receiving their test results. Families with a lower incidence of CRC and extracolonic cancers, in the face of being positive for Amsterdam I criteria but who do not have MMR deficiency by tumor testing, are probably not Lynch syndrome, and thereby should preferably be designated as familial CRC of undetermined type. Patients who are either noncompliant or poorly compliant with colonoscopy, and who are MMR mutation positive, may be candidates for prophylactic colectomy, while MMR mutation-positive women who are noncompliant with gynecologic surveillance may be candidates for prophylactic hysterectomy and bilateral salpingo-oophorectomy. European Journal of Human Genetics (2006) 14, 390-402. doi:10.1038/sj.ejhg.5201584; published online 15 February 2006

Keywords: colorectal cancer; hereditary cancer; cancer genetics; Lynch syndrome; HNPCC

\section{Introduction}

Colorectal cancer (CRC) poses a significant problem throughout the world as reflected by its annual worldwide incidence of over one million $(1023152) .{ }^{1}$ It is estimated that approximately $15 \%$ (153473) of these CRC cases are familial and that an additional 10\% (102315) are

*Correspondence: Dr HT Lynch, Department of Preventive Medicine, Creighton University School of Medicine, 2500 California Plaza, Omaha, NE 68178, USA. Tel: +1 402280 2942; Fax: +1 402280 1734;

E-mail: htlynch@creighton.edu

Received 12 July 2005; revised 22 November 2005; accepted 28 December 2005; published online 15 February 2006 hereditary. Worldwide, Lynch syndrome, also referred to as hereditary nonpolyposis CRC (HNPCC) is the most common hereditary variant of CRC, accounting for $>2 \%(20463)$ and probably $<5 \%$ (51 158) of new cases annually. ${ }^{2,3}$

Lynch syndrome is characterized by early age at onset of CRC, excess of synchronous and metachronous CRCs, a predilection to the proximal colon, accelerated carcinogenesis, and an excess risk for extracolonic cancers (including endometrium and, to a lesser extent, ovary, stomach, hepatobiliary tract, small bowel, pancreas, brain, transitional cell carcinoma of the ureter and renal pelvis, and, in the Muir-Torre syndrome variant of the Lynch syndrome, 
sebaceous tumors. ${ }^{2,3}$ Pathology findings in the Lynch syndrome include tumor-infiltrating lymphocytes, signet ring cells, and a strong mucinous component. ${ }^{4,5}$ Figure 1 shows the pedigree of a Lynch syndrome family (Muir-Torre syndrome variant) displaying an array of extracolonic cancers.

During the past decade, advances in molecular genetics have provided new insights into the etiology of a number of hereditary gastrointestinal cancers, enabling greater precision in hereditary cancer syndrome identification. ${ }^{6}$ Because of the complexity of hereditary cancer syndromes, the challenge to the clinician is the need to understand the natural history of hereditary cancer syndromes and their molecular genetics, which together can improve diagnosis, surveillance, and management strategies for cancer control. To effectively accomplish this, a detailed family history is needed, together with genetic counseling., ${ }^{2,3}$

First of all, it is important to understand that hereditary disorders of the gastrointestinal tract are not rare. Familial adenomatous polyposis (FAP) accounts for much $<1 \%$ of the total CRC burden, in comparison to the more prevalent Lynch syndrome (Figure 2). Insufficiently detailed collection of family history and/or limited knowledge of syndrome phenotype contribute to nonrecognition of affected patients and families. The presence of a deleterious germline mutation in the patient/family will enable a high level of diagnostic certainty in hereditary CRC syndromes such as FAP with its APC mutation, and the Lynch syndrome with mutations in mismatch repair (MMR) genes (MSH2, MLH1, MSH6, PMS2).

Molecular genetic heterogeneity must be considered when assessing the Lynch syndrome cancer phenotype. While more evidence will be required to substantiate differences in phenotype, current data suggest that $M L H 1$ mutations have a higher expression of CRC than do MSH2 mutations, which have more extracolonic cancers and Muir-Torre features. MSH6 mutations, when compared with those of MLH1 and MSH2, show a lower expression of CRC but an excess of endometrial cancer. The phenotypic consequences of PMS2 mutations appear to be highly variable, often with childhood onset of atypical tumors, but the number of observations is too limited for firm conclusions at this time.

\section{Common mutations}

Mutations that are widespread can be either recurrent, that is occurring repeatedly de novo, or ancestral (founder mutations), having occurred once and passed on to numerous progeny. The best-known recurrent mutation

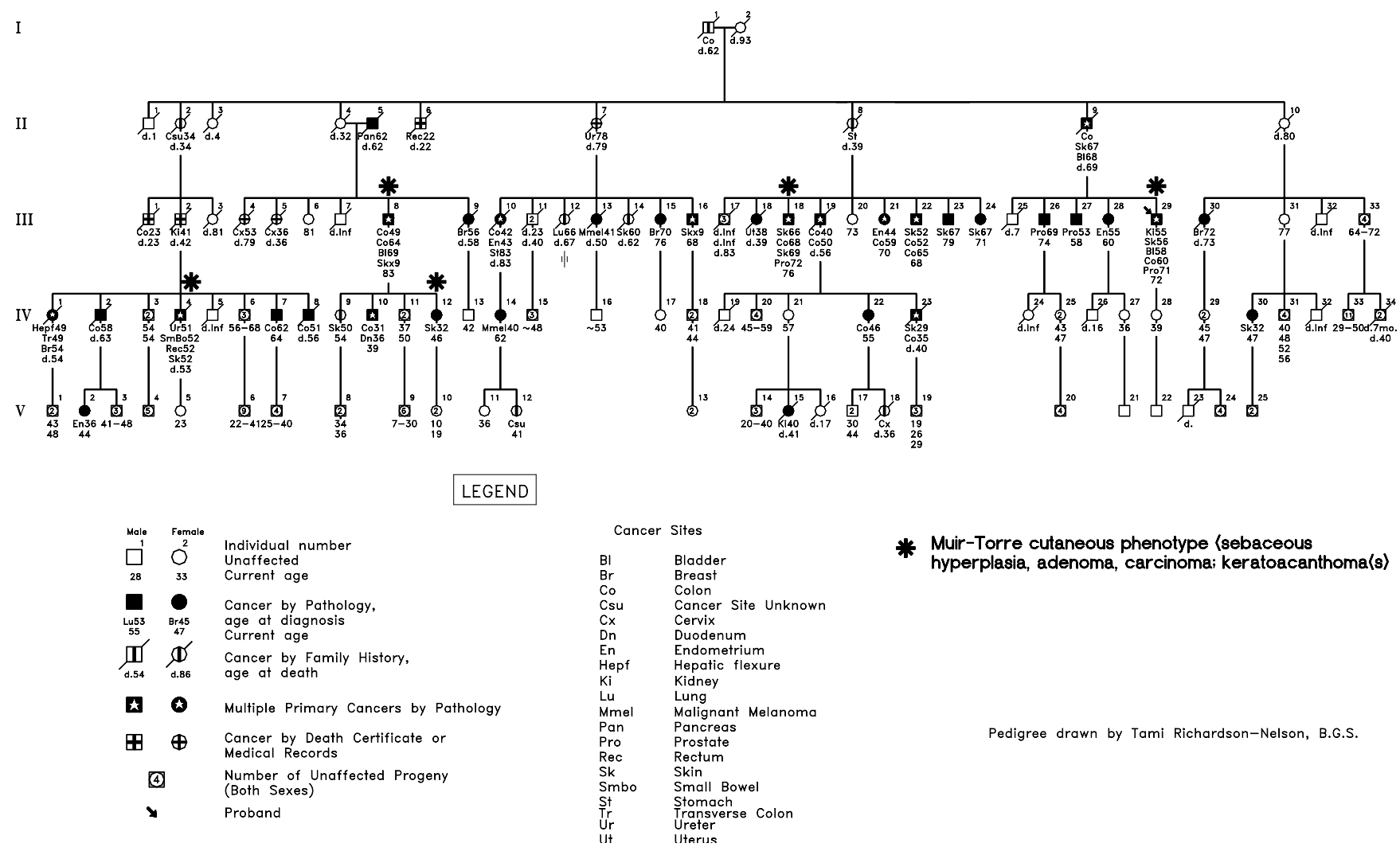

Figure 1 Pedigree of Muir-Torre family with multiple extracolonic cancers (reprinted from Lynch et al. Am J Gastroenterol 2003; 98: 664-670). 


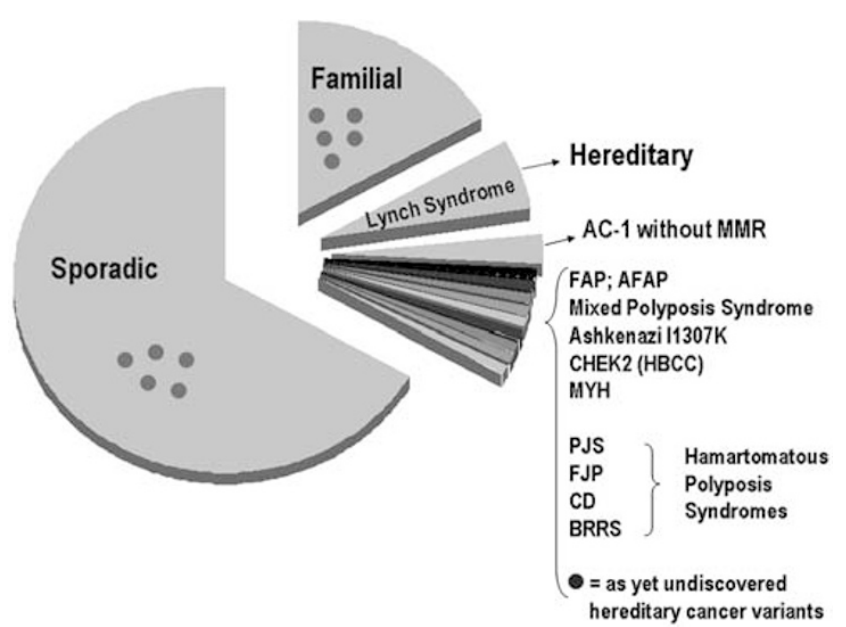

Figure 2 Circle graph showing relative numbers of colorectal cancer cases that are considered sporadic, familial, or due to a recognized hereditary cancer syndrome. (Updated with permission from Lynch et al. Cancer 2004; 100: 53-64.) MMR = mismatch repair; $\mathrm{FAP}=$ familial adenomatous polyposis; $\mathrm{AFAP}=$ attenuated familial adenomatous polyposis; $\mathrm{HBCC}=$ hereditary breast and colorectal cancer; PJS $=$ Peutz-Jeghers syndrome; $F J P=$ familial juvenile polyposis; $C D=$ Cowden's disease; $B R R S=$ Bannayan - Ruvalcaba-Riley syndrome.

causing Lynch syndrome is an $\mathrm{A} \rightarrow \mathrm{T}$ transversion in the donor splice site of intron 5 of $\mathrm{MSH} 2$ (designation c. $942+3 \mathrm{~A} \rightarrow \mathrm{T}){ }^{7,8}$ This mutation apparently arises repeatedly because the region is vulnerable to slippage during the DNA replication process, the mutated nucleotide being the first in a microsatellite consisting of 26 adenines. It occurs worldwide ${ }^{9}$ and may account for as much as $5-10 \%$ of all Lynch syndrome. ${ }^{10}$

The ability of an ancestral mutation to persist and become widespread in a population even though it causes a serious condition such as Lynch syndrome (lethal in the era before surgery) is explained by the fact that reproduction is not significantly affected. Thus, some Lynch syndrome founder mutations have become quite common in typical founder populations. In Finns, a genomic deletion of exon 16 of MLH1 probably dates back 1000 years or more and in parts of Finland accounts for $>50 \%$ of all Lynch syndrome. ${ }^{11,12}$ Other highly enriched founder mutations have been documented in Ashkenazi Jews (MSH2 1906G $\rightarrow$ C; accounting for $\sim 20 \%$ of Lynch syndrome ${ }^{13}$ ) and other genetically isolated populations (for review see de la Chapelle ${ }^{14}$ ).

Founder mutations usually occur in isolated populations that favor enrichment through bottlenecks and genetic drift. The existence of a founder mutation in the general US population was not expected; but in fact one such mutation was uncovered very recently, ${ }^{15,16}$ namely, a deletion of exons 1-6 of MSH2. This mutation had remained undiscovered because it was not detectable by standard methods such as sequencing.
This exon 1-6 deletion in MSH2 was originally detected in nine apparently unrelated multigenerational kindreds with Lynch syndrome, ${ }^{17}$ and has subsequently been identified in 13 more families (Lynch, unpublished data, 2005). The sequence of the breakpoints of this exon 1-6 deletion and the haplotypes surrounding the mutation were identical in all nine kindreds, ${ }^{15,17,18}$ suggesting a common origin of the mutation. It was subsequently traced through three of the original nine kindreds by genealogy studies through 11-12 generations to a putative common founder who migrated from Germany to the US in $1727 .{ }^{17}$ Based on our pedigrees and published epidemiology data, we estimate that there are at least 19000 expected living AFM carriers in the United States. ${ }^{19}$ The original nine families with the AFM account for 13\% (9/70) of families with Lynch syndrome that had been tested and found to be 'negative' for MMR mutations by conventional mutation screening techniques. The high percentage of this founder mutation among Lynch syndrome cases bears significant implications for cancer control. Testing for recurrent and founder mutations as a first step may considerably facilitate the molecular diagnosis of the disease in the appropriate populations.

\section{Children with homozygous $\mathrm{MLH1}$ mutation}

The carrier rate for heterozygous mutations in MMR genes in Lynch syndrome remains elusive. However, the marriage of two carriers would lead to the probability that one in four of their offspring would be homozygous for the mutation. Homozygous and compound heterozygous MMR gene mutations in children had formerly been described as predisposing to leukemia, lymphoma, and brain tumors, and café au lait spots in the absence of gastrointestinal carcinomas. However, Gallinger et $a l^{20}$ have reported the first known instance of children with homozygous MMR gene deficiency manifesting early-onset gastrointestinal cancers in concert with neurofibromatosis type 1 (NF1) features. All three members of one sibship '... had a germline homozygous MLH1 missense mutation (exon 18, codon 687, CGGTGG), whereas both parents were heterozygous for this mutation.' It is noted that any progeny from two heterozygous MMR mutation carriers will have a one-in-four chance of being compound heterozygotes or true homozygotes, in which case they would be likely to manifest a stronger predisposition to cancer than would be the case for those who are heterozygotes. It is worth noting that these individuals are extremely rare.

\section{Modifier genes}

Interindividual variations in the genome can be unrelated to disease, especially when they occur outside of genes. In contrast, variations in genes, especially in the coding regions, have the potential of causing phenotypic 
consequences such as a predisposition to cancer. Such effects are often subtle, hence the description low penetrance genes, modifier genes, risk alleles. In colon cancer, the prime example of a low-penetrance allele is the $11307 \mathrm{~K}$ polymorphism in the $A P C$ gene that occurs in $6-7 \%$ of Ashkenazi Jews. ${ }^{21}$ It confers an approximately two-fold elevated risk of CRC by promoting genomic instability and frameshift mutations in the immediate genomic vicinity of codon 1307. Interestingly, even though the risk is quite modest, it can be calculated that as many as $5-7 \%$ of all CRC in Ashkenazi Jews is caused by this variant. Another variant, $T G F B R 1{ }^{*} 6 A$ occurs in approximately $14 \%$ of Caucasians and conveys a very modest $\sim 1.2$-fold risk of CRC, yet this may underlie $3 \%$ of all CRC. ${ }^{14}$ The * $6 \mathrm{~A}$ risk allele in TGFRB1 was recently shown to be much more prevalent in those Amsterdam criteria-positive CRC patients who did not have a MMR gene mutation (allele frequency 0.195) than in those with a MMR gene mutation $(0.104) .{ }^{22}$ It follows that this allele may cause or help cause non-Lynch syndrome hereditary CRC. This allele may be extremely important not only when present in the germ line, but also as a somatically acquired change highly associated with CRC metastases to the liver. ${ }^{23}$

Recently, it was proposed that the well-known polymorphism in codon 72 of the TP53 gene has a profound effect on the age of onset of CRC in Lynch syndrome, ${ }^{24}$ and the findings were confirmed by Kruger et al. ${ }^{25}$ However, in a third study, ${ }^{26}$ no such effect was seen. This illustrates the fact that genetic association studies are, especially whenever the studied effect is relatively subtle, difficult to interpret and often contradictory.

\section{Microsatellite instability (MSI) and immuno- histochemistry (IHC) screening}

Vasen and Boland ${ }^{27}$ note that MSI in CRC was discovered in $1993,{ }^{28,29}$ (cited by Vasen and Boland) and subsequently it was found that MSI was present in colon cancer tissue from most Lynch syndrome patients ${ }^{30-32}$ (cited by Vasen and Boland). However, the molecular genetic sine qua non for Lynch syndrome diagnosis is a germline mutation in one of the MMR genes. The major MMR genes that harbor causal mutations for Lynch syndrome are MSH2, MLH1, MSH6 and PMS2. Individuals with such mutations have an $\approx 70 \%-85 \%$ risk, based on the mutation's penetrance, to develop CRC by the age of 60 years. ${ }^{33}$ A subset of MMR germline mutations involving large deletions of entire exons are often unidentifiable using regular testing techniques. However, they can be found through various alternative techniques such as those used to identify the already-mentioned MSH2 exon 1-6 deletion. ${ }^{15,17}$

From the standpoint of the clinician, it is important to consider the role of MSI and immunohistochemical (IHC) staining, either separately or in combination, as intermediate instruments of screening for the Lynch syndrome. ${ }^{34}$
Should all CRC-affected individuals be tested? According to one school of thought, the answer is yes. ${ }^{10}$ It would be costly and, therefore, should be tested in larger clinical trials, for example, using IHC as the primary screen, to determine if it leads to considerable saving. Thus, the cost effectiveness could be determined more precisely before any general recommendations would be made. ${ }^{10,34}$ MSI and loss of expression of MLH1 by IHC in tumors of older subjects is more likely to be related to hypermethylation of the MLH1 promoter and thereby will carry a very low positive predictive value for the Lynch syndrome, particularly when present in the absence of a family history targeting the syndrome. An assay for methylation of the MLH1 promoter, is a relatively simple way of determining whether MSI or loss of the MLH1 protein on IHC staining is likely (no methylation) or unlikely (methylation) to signal Lynch syndrome.

In discussing the strategy for using MSI and IHC molecular markers, Vasen and Boland ${ }^{27}$ suggest that IHC may aid in the search for mutations to a specific gene, while in those patients who meet the Bethesda guidelines they suggest that the first step might be MSI analysis followed by IHC of all tumors classified as MSI-high $(\mathrm{MSI}-\mathrm{H})^{35}$ (cited by Vasen and Boland). While recognizing that MSI and IHC have been available for only a very short time, Vasen and Boland also urge that every effort be made to extend the current small-scale use of these molecular diagnostic tools in order to make them available to all clinicians. However, in order for this effort to be successful, clinicians must become familiar with the pros and cons of these tests as well as the Bethesda guidelines (Table 1). ${ }^{27}$

Two major questions arise, first, should Lynch syndrome be searched for more actively than hitherto, and second, what should be the primary screening method? Family history is regrettably often ignored ${ }^{36}$; moreover, both family history and age are losing their sensitivity to detect Lynch syndrome as family size decreases and the more atypical phenotypes associated with MSH6 and PMS2 mutations are taken into account. In the study of unselected CRC patients in Ohio, 23/1066 (2.2\%) had Lynch syndrome. ${ }^{10}$ Importantly, only $3 / 23$ patients fulfilled the Amsterdam criteria; 5/23 patients did not even fulfill the Bethesda guidelines. A total of 10 of 23 patients were over the age of 50 years at diagnosis; five of these were over 60 years. These facts argue strongly against relying on family history and age alone if one wishes to diagnose most cases of Lynch syndrome.

IHC is emerging as the method of choice for primary screening. In the Hampel et $a l^{10}$ study both IHC and MSI each failed to detect $2 / 23$ patients with Lynch syndrome, that is, both methods showed a sensitivity of $>90 \%$. Similar results based on high-risk patients were shown by Lindor et al. ${ }^{37}$ To assess definitively the sensitivity of IHC one should test all cases by all methods (IHC, MSI, mutation detection) and compare the results. This cannot 
Table 1 Amsterdam I and Amsterdam II criteria, and Bethesda guidelines

Amsterdam I criteria ${ }^{80}$ :

- At least three relatives with histologically verified colorectal cancer:

1. One is a first-degree relative of the other two;

2. At least two successive generations affected;

3. At least one of the relatives with colorectal cancer diagnosed at $<50$ years of age;

4. Familial adenomatous polyposis has been excluded.

Amsterdam // criteria $^{81}$ :

- At least three relatives with an hereditary nonpolyposis colorectal cancer-associated cancer (colorectal cancer, endometrial, stomach, ovary, ureter/renal pelvis, brain, small bowel, hepatobiliary tract and skin (sebaceous tumors)):

1. One is a first-degree relative of the other two;

2. At least two successive generations affected;

3. At least one of the hereditary nonpolyposis colorectal cancer-associated cancers should be diagnosed at $<50$ years of age;

4. Familial adenomatous polyposis should be excluded in any colorectal cancer cases;

Tumors should be verified whenever possible.

Bethesda guidelines for testing of colorectal tumors for microsatellite instability ${ }^{82}$ :

1. Colorectal cancer diagnosed in a patient who is $<50$ years of age.

2. Presence of synchronous or metachronous colorectal, or other HNPCC-associated tumors, ${ }^{a}$ regardless of age.

3. Colorectal cancer with the MSI- $\mathrm{H}^{\mathrm{b}}$ histology ${ }^{\mathrm{c}}$ diagnosed in a patient who is $<60$ years of age. ${ }^{d}$

4. Colorectal cancer or HNPCC-associated tumor ${ }^{a}$ diagnosed under age 50 years in at least one first-degree relative. ${ }^{\text {e }}$

5. Colorectal cancer or HNPCC-associated tumor ${ }^{a}$ diagnosed at any age in two first- or second-degree relatives. ${ }^{\mathrm{e}}$

${ }^{a}$ Hereditary nonpolyposis colorectal cancer (HNPCC)-associated tumors include colorectal, endometrial, stomach, ovarian, pancreas, ureter or renal pelvis, biliary tract, and brain (usually glioblastoma as seen in Turcot syndrome) tumors, sebaceous gland adenomas and keratoacanthomas in Muir-Torre syndrome, and carcinoma of the small bowel.

${ }^{\mathrm{b}} \mathrm{MSI}-\mathrm{H}=$ microsatellite instability-high in tumors refers to changes in two or more of the five National Cancer Institute-recommended panels of microsatellite markers.

'Presence of tumor-infiltrating lymphocytes, Crohn's disease-like lymphocytic reaction, mucinous/signet-ring differentiation, or medullary growth pattern.

dThere was no consensus among the Workshop participants on whether to include the age criteria in guideline 3 above; participants voted to keep $<60$ years of age in the guidelines.

${ }^{\mathrm{e}}$ Criterias 4 and 5 have been reworded to clarify the Revised Bethesda Guidelines.

be done at reasonable cost. In a study of 1222 unselected CRC patients from Spain, Piñol et $a l^{38}$ did test all for IHC and MSI and found an excellent correlation between the two. This argues in favor of the much simpler IHC; however, it is unclear why in this study so few patients had abnormal IHC/MSI (6\%) and so few had Lynch syndrome $(0.7 \%)$. As a conclusion, we take the position that IHC loss of protein expression is the most desirable test to screen for Lynch syndrome.

\section{Genetic/environmental interaction}

Cancer-causing mutations do not act in a vacuum; rather, environmental perturbations may play an extremely important role in modifying molecular genetic susceptibility to cancer. Yet, there has been little information about possible environmental modulation of cancer phenotype in the Lynch syndrome.

Watson et $\mathrm{al}^{39}$ evaluated tobacco use and the manner in which it might alter CRC expression in carriers of Lynch syndrome-associated mutations, using a retrospective cohort study of MLH1 and MSH2 mutation carriers from Creighton University's Hereditary Cancer Institute. Results showed that MLH1 mutation carriage (as opposed to MSH2) and male sex were significantly associated with increased risk of CRC (hazard ratios, 1.43, 2.07 and 1.58, respectively). Alcohol consumption did not alter the CRC risk. It was concluded that smoking cessation should be an integral component of Lynch syndrome management. These data underscore the gene $\times$ environmental interactions in CRC development in the Lynch syndrome.

\section{Genetic counseling}

It is important that genetic counseling take place prior to DNA collection and testing, as well as at the time of disclosure of results, since the presence of a cancer-causing germline mutation may impact heavily on the patient's emotions when dealing with his or her lifetime destiny for cancer. Thus, this precious and potentially lifesaving knowledge will enable disclosure of risk for cancer(s) of specific anatomic sites, their average age of onset, prognostic implications, and possibly even chemotherapy choices $^{40-49}$ (discussed subsequently), in the patient bearing the specific cancer-causing mutation. This knowledge will be limited only by the deleterious mutation's penetrance and the mentioned potential impact of environmental carcinogenic interaction.

Counselors must be prepared to discuss patients' psychological concerns and thereby address freely such issues as survivor guilt if the patient is found to be negative for the deleterious mutation, or, if positive, concerns about insurance and employment discrimination. Surveillance and management must be addressed, in the interest of compliance with these potentially life-saving measures, all in context with possible fear, anxiety, and apprehension about his or her long-term cancer destiny.

\section{Family information session (FIS)}

Cancer control and sound psychological objectives can be significantly abetted through a FIS. ${ }^{50}$ This involves the participation of as many family members as desire to assemble at an educational session, in a geographic area of convenience to the family. The FIS is conducted by a physician, a study coordinator who may be a registered 
nurse, and a genetic counselor. Participants are educated in depth about the natural history of the particular hereditary cancer syndrome in their family, availability of genetic testing, and the surveillance and management options available to them. Blood draws for germline mutation testing, when indicated, will follow genetic counseling on consenting individuals.

These FIS sessions enable the physician and genetic counselor to discuss diagnostic and cancer control measures, as well as help to define those confidentiality, psychosocial and economic concerns that may impact heavily upon the family, and then determine how best to resolve them. A group form of psychosocial therapy often evolves. Family members will frequently state that this was the first time in their lives that a physician told them 'face to face' what could kill them and, in turn, what they could do about ameliorating the many diagnostic, surveillance, and management problems that they may encounter and/ or may have already encountered as a result of being at increased hereditary cancer risk.

\section{Confidentiality issues}

One of the thorniest issues in genetic counseling pertains to the role of confidentiality and the extent of disclosure of cancer risk information, such as the presence of a cancercausing germline mutation of the APC gene in FAP, or of an MMR gene in the Lynch syndrome, to an affected individual's close at-risk relatives. This disclosure may be particularly problematic when that gene carrier is strongly opposed to disseminating this information to at-risk relatives. Thus, we see an immediate conflict with respect to the duty of the physician/genetic counselor to maintain confidentiality in concert with the duty to warn.

A subcommittee of the American Society of Human Genetics dealing with family disclosure, ${ }^{51}$ has suggested that ethical, legal, and statutory exceptions, '...limit the principle of confidentiality and, in specific and very limited circumstances, may permit disclosure. At the very least, it is clear that a healthcare professional has a positive duty to inform a patient about potential genetic risks to the patient's relatives...' Where certain preventive action can be taken, the duty to warn becomes of compelling concern.

\section{$\mathrm{MSI} / \mathrm{IHC}$ and recognition of Lynch syndrome: standards of care upgraded?}

It is our premise that when a patient presents with CRC, the endoscopist, surgeon, and oncologist have the responsibility to recognize the possibility that an hereditary predisposition may be present. Most cases will sort themselves out as clearly hereditary (diffuse polyposis at an early age) or clearly sporadic (an elderly patient with no family history of malignancy). In between lies the challenging world of possible Lynch syndrome. In this syndrome, early age of onset has been ingrained in the literature, although Hampel et $a l^{52}$ as mentioned, have called appropriate attention to older onset (55-60 years).

The presence of major clues such as fulfillment of the Amsterdam criteria, very early age at onset, multiple primary cancers that are integral to Lynch syndrome, and/or the presence of key pathologic features, collectively may provide a basis for MMR gene testing. However, because the sensitivity of mutational testing is low when predicated on these features, any measure that can be employed to increase the specificity of mutational testing without compromising sensitivity should be considered. The predictive power of MSI and/or IHC for loss of MMR gene expression must be considered and have been found to be increasingly helpful in this regard. ${ }^{27}$ Several large series have now shown that the yield of mutational testing is low when a tumor is microsatellite stable, even when the family history is otherwise very suggestive. Conversely, with the exception of methylation-mediated silencing of MLH1, which can lead to a 'false positive' tumor screen, MSI and IHC serve as reliable predictors of an underlying Lynch syndrome mutation, even when the family history is soft or the patient is older. For these reasons, our threshold for conducting MSI/IHC has become rather low. ${ }^{10}$

\section{Amsterdam criteria positive but MMR-negative cohort}

Lindor et al $^{53}$ described 3422 relatives from 161 Amsterdam criteria I (AC-1) families and divided them via tumor testing into group A (with MMR deficiency) and group B (without MMR deficiency. Group A families showed an increased incidence of Lynch syndrome-related cancers while group B families showed an increase only for CRC, and even then to a lesser extent than group $\mathrm{A}$. It was concluded that families who fulfill AC-1 criteria but lack evidence of DNA MMR defect do not show the same cancer incidence as families with Lynch syndrome and an MMR deficiency. Furthermore, those relatives in group B families also showed a lower incidence of those extracolonic cancers integral to Lynch syndrome when compared with their group A counterparts. These authors suggest that families in the group B category '... should not be described or counseled as having HNPCC-Lynch syndrome.' To facilitate distinguishing these entities, the designation of 'familial CRC type $\mathrm{X}^{\prime}$ was suggested to describe this type of familial aggregation of CRC. ${ }^{53}$ However, to avoid confusion with $\mathrm{X}$ chromosomal inheritance we prefer the designation 'familial CRC of undetermined type.' We advocate surveillance of these high-risk patients comparable to that for the Lynch syndrome.

It is also pertinent to note that Lynch et al ${ }^{54,55}$ had previously classified families into Lynch syndrome I, which was characterized by families with increased CRC risk but lacking associated extracolonic cancers, and Lynch 
syndrome II, which contained the associated integral cancers in addition to CRC excess. These studies took place in the 1980s, prior to knowledge about MMR mutations and their causal significance in the Lynch syndrome.

\section{Cardinal features of Lynch syndrome}

- Earlier average age of CRC onset than in the general population; the average age of CRC onset in Lynch syndrome is $\approx 45$ years, Hampel et al ${ }^{52}$ reporting 55-60 years of age in all Finnish Lynch syndrome families, while the average age of onset in sporadic CRC is $\approx 65$ years;

- Proximal colon involvement (70\% of CRCs arise proximal to the splenic flexure);

- A significant excess of synchronous and metachronous CRCs $(\approx 25-30 \%$ of patients having a second primary CRC within 10 years of surgical resection for initial CRC, if the surgery was anything less than a subtotal colectomy);

- Autosomal dominant inheritance pattern;

- Increased risk for malignancy at certain extracolonic sites, foremost of which is endometrial carcinoma, followed by carcinoma of the ovary, stomach, small bowel, hepatobiliary tract, pancreas, upper uro-epithelial tract and brain; putative Lynch syndrome cancers lacking sufficient evidence to be unconditionally added to the list include adrenal cortical carcinoma, ${ }^{56}$ sarcomas (malignant fibrous histiocytoma, ${ }^{57}$ rhabdomyosarcoma, ${ }^{58}$ liposarcoma ${ }^{59,60}$ ) prostate cancer, ${ }^{61}$ and patients with neurofibromatosis features. ${ }^{20}$

- CRC tumors in Lynch syndrome are more often poorly differentiated, with an excess of mucoid and signet-cell features, show a Crohn's-like reaction, and contain a significant excess of infiltrating lymphocytes within the tumor. MSI is found in most CRC tumors in Lynch syndrome;

- Increased survival from CRC, when controlled for age and stage;

- Accelerated carcinogenesis and interval CRC; a tiny adenoma may emerge into a carcinoma within 2-3 years, as opposed to 8-10 years in the general population;

- Sebaceous adenomas, sebaceous carcinomas, and multiple keratoacanthomas in the Muir-Torre syndrome variant of Lynch syndrome;

- The sine qua non, the identification of a germline MMR mutation segregating with syndrome-affected individuals in the family.

\section{Cancer control in Lynch syndrome}

Cancer-related morbidity and mortality may be reduced significantly through highly targeted surveillance and management measures that are based on knowledge of the natural history and cardinal features of the Lynch syndrome. Particularly important is full colonoscopy (due to the syndrome's predilection toward proximal CRC), ${ }^{62}$ initiated at the age of 25 years (due to early onset of CRC), ${ }^{3}$ with good cleanout for visualization to the cecum, and repeated annually (due to accelerated carcinogenesis). ${ }^{4,63}$

For women, endometrial aspiration coupled with transvaginal ultrasound is advised for screening, beginning at the age of 30-35 years. We recommend semiannual CA 125 testing, beginning at the same age, for ovarian cancer. Women must be advised of the marked limitations in ovarian cancer screening. ${ }^{64}$ Prophylactic hysterectomy and oophorectomy can then be considered when childbearing is completed.

These cancer control strategies impact heavily upon at-risk family members once they have been counseled and educated thoroughly about Lynch syndrome's natural history and their own hereditary cancer risk. Figure 3 is an algorithm showing proposed steps in the diagnosis and management of Lynch syndrome patients.

\section{Does colonoscopy work?}

Järvinen et $a l^{65}$ demonstrated the lifesaving benefit of colonoscopic surveillance in Lynch syndrome through a controlled clinical trial extending over 15 years. The incidence of CRC was compared in two cohorts (screened and nonscreened) of at-risk members of 22 Lynch syndrome families. The nonscreened (controls) were family members who were noncompliant with respect to colonoscopy. CRC developed in eight screened subjects $(6 \%)$, compared with 19 unscreened controls $(16 \% ; P=0.014)$. The CRC rate was reduced by $62 \%$ in those who were screened. All CRCs in the screened group were local, causing no deaths, compared with nine deaths caused by CRC in the controls. It was concluded that CRC surveillance at 3-year intervals more than cuts in half the CRC risk, prevents CRC deaths, and decreases overall mortality by about $65 \%$ in Lynch syndrome families. The relatively high incidence of CRC even in the screened subjects (albeit without deaths) argues for shorter intervals between colonoscopies, for example, 1 year. For example, Vasen et al $^{66}$ discovered five cancers in Lynch syndrome patients within a $3 \frac{1}{2}$ years interval following a normal colonoscopy.

\section{Prophylactic colectomy}

Subtotal colectomy as a prophylactic measure among Lynch syndrome patients remains controversial. However, in special circumstances, we propose that patients who carry germline MMR cancer-causing mutations, or who develop an early colonic adenoma, should be offered this option as an alternative to lifetime colonoscopic surveillance. Church ${ }^{67}$ and Lynch $^{68}$ have suggested that a special need exists for the option of prophylactic surgery for those patients likely to show reduced compliance for 
Part A

Lynch Syndrome: Diagnosis and Management

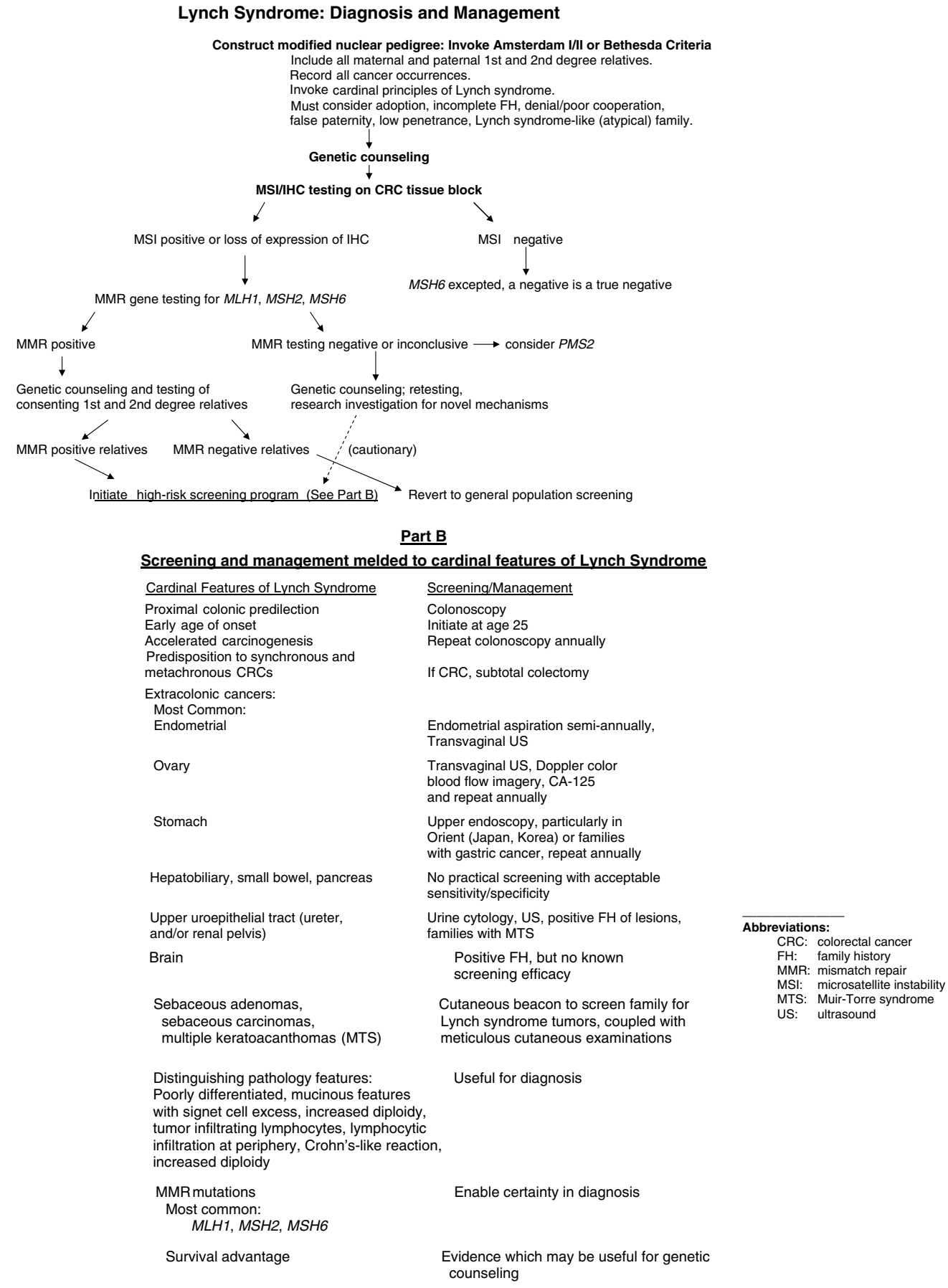

Figure 3 Algorithm showing proposed diagnosis and management procedures for Lynch syndrome families. The screening and management guidelines shown are the recommendations of the authors, rather than being evidence based.

colonoscopy and/or poor quality of life due to fear and anxiety about the threat of CRC due to their knowledge that they harbor an MMR mutation. Genetic counseling, coupled with a second surgical opinion, must be provided so that patients can evaluate the various available surgical management strategies. Given the high risk for metachronous CRC in Lynch syndrome patients, we recommend subtotal colectomy for initial CRC, assuming that the patient's CRC can be controlled. ${ }^{2,3}$ The remaining rectal segment should be screened annually. 


\section{Chemotherapy concerns}

The absence of a positive response to 5-fluorouracil (5-FU) in Lynch syndrome

In 1993, Parsons et al $^{69}$ reported that the colon cancer cell line HCT116 had MSI, and an extract from these cells failed to repair mismatched DNA heteroduplexes. It was later shown that the MLH1 gene on chromosome $3 p$ was homozygously mutated in these cells. In 1994, Koi et al derived a cell line from HCT116 by stably transferring another copy of chromosome 3 into the parental line. The cell line, HCT116+ch3, expressed MLH1 mRNA and protein, had a restoration of DNA MMR activity, and the microsatellite stability was corrected. Importantly, restoration of the DNA MMR activity reduced the tolerance of the tumor cells to the toxic alkylating agent, MNNG. The group subsequently demonstrated that correction of the DNA MMR system also restored the G2/M cell cycle checkpoint to the tumor cells. Thus, critical degrees of DNA damage, induced either by MNNG or 6-thioguanine, would induce G2/M cell cycle arrest in the DNA MMRproficient cell line, whereas the parental HCT116 cell line tolerated this challenge, and presumably, would be susceptible to the accumulation of an additional mutational load if challenged. ${ }^{71}$ Carethers et $a l^{72}$ then found that competency of the DNA MMR system prohibited clonal expansion of cancer cells after exposure to MNNG, using a panel of cell lines that were DNA MMR proficient (such as SW480 or HCT116+ch3). Contrariwise, the cloning efficiency after DNA damage was significantly higher in the DNA MMR-deficient cell lines HCT116, the chromosome 2 transferred line HCT116 + ch2 (which served as a control for chromosome 3 transfer), the cell line HCT116+ ch3M2 (in which the MLH1 gene had been inactivated by mutation from HCT116 + ch3 cells), as well as cell lines LoVo and 2774. The observation that an abnormality in DNA MMR conferred resistance to DNA damage had been made by Branch et al ${ }^{73-75}$ in non-colonic cells even before the human DNA MMR genes had been identified or linked to Lynch syndrome.

These observations established that the DNA MMR system participated in the cell death response to DNA damage, which is the principal mechanism by which cancer chemotherapy kills cancer cells. In order to determine whether a similar response would be found using 5-FU, colony forming activity in the presence of this drug was examined in MMR-deficient and MMR-proficient cell lines. All of the cell lines with defective DNA MMR activity, whether due to defective MLH1 activity (HCT116) or defective MSH2 activity (LoVo, 2774), were more tolerant of doses of 5 -FU up to $5 \mu \mathrm{M}$. Contrariwise, the DNA MMR-corrected cell line HCT116 + ch3, and the CRC cell line SW480, which is DNA MMR proficient, showed a dose-related fall in colony-forming activity after exposure to 5-FU. Importantly, this dose of 5-FU is pharmacologically relevant, and suggested that clinical resistance to 5-FU might be mediated, at least in part, by defective DNA MMR activity. Arnold et $a l^{40}$ also demonstrated that reversal of silencing of the MLH1 gene in SW48 cells by the demethylating agent, 5-azacytidine, also restores sensitivity to the toxic effects of 5-FU.

This approach was extended by Aebi et al, ${ }^{41}$ in which the DNA MMR-deficient cell lines HCT116 and HEC59 (deficient in MSH2) were two-fold resistant to cisplatin, compared with DNA MMR-proficient cells, including both HCT116+ch3 and HEC59+ch2, in which the MSH2 deficiency was corrected. Thus, in vitro laboratory experimentation predicted that cancers with defective DNA MMR activity and MSI might be resistant to many commonly used chemotherapeutic agents, and in the case of 5-FU, the most commonly used agent, this was true in the pharmacologically relevant range.

Interestingly, Magrini et $a l^{42}$ showed that the absence of MLH1 protein actually enhances CPT-11-induced apoptosis, and that triggering of cell cycle arrest was p53 independent. Thus, a theoretical basis was formed for the development of chemotherapeutic strategies specifically targeted to $\mathrm{MSI}^{+}$cells.

Meanwhile, several groups had demonstrated that young patients with $\mathrm{MSI}^{+}$CRCs have a favorable natural history compared to those with microsatellite stable cancers. ${ }^{43}$ Because these patients were under 50 years of age, most were thought to have Lynch syndrome. In fact, it was the failure to take the MSI factor into account properly that led to the spurious report that a survival benefit had been achieved in a retrospective study of adjuvant chemotherapy in patients with $\mathrm{MSI}^{+} \mathrm{CRCs}^{76}$ Precisely the opposite was actually true. The fatal flaw of this study was the use of a retrospective study design, and the failure to randomize the patients prior to selection for chemotherapy. Because patients with Lynch syndrome tend to be substantially younger and apparently healthier than those with sporadic colon cancer, there is a tendency to preferentially select these patients for adjuvant chemotherapy. Subsequently, it was demonstrated in a large, prospective, randomized study of chemotherapy that 5-FU was of benefit to patients with CRCs that were microsatellite stable, but a modest, two-fold hazard ratio for death was found among those patients who received 5-FU if their tumor was $\mathrm{MSI}^{+} .{ }^{44}$ The issue of survival will confound studies that are not prospectively randomized and stratified for MSI status. Nonetheless, a failure to demonstrate survival benefits after adjuvant chemotherapy in patients with $\mathrm{MSI}^{+}$tumors or with Lynch syndrome has been demonstrated by most subsequent investigators. ${ }^{45-48}$

Fortunately, newer options are available for treating patients with $\mathrm{MSI}^{+}$CRCs, including patients with Lynch syndrome. Fallik et al $^{49}$ performed a retrospective analysis of 72 patients with metastatic CRC, and demonstrated that patients with $\mathrm{MSI}^{+}$tumors had a better response to the topoisomerase I inhibitor, irinotecan. Thus, it is reasonable 
to consider the planning of future clinical trials in which non-5-FU-based chemotherapy will be used in the adjuvant setting for patients with Lynch syndrome, as well as for other patients with advanced $\mathrm{MSI}^{+}$cancers. Also, as there are important biological differences between Lynch syndrome tumors and sporadic $\mathrm{MSI}^{+}$tumors (which are caused by the epigenetic silencing of MLH1 and other tumor suppressor genes), these groups may need to be considered separately. ${ }^{48}$

\section{Discussion and summary}

Can generalizations be drawn regarding the approach to the next clinic patient who enters your clinic? The answer is a qualified yes, so let us begin. The history and physical may provide clues. Family history may point to the presence of Amsterdam criteria. For better or worse, even when the patient is familiar with details of his or her family history, most cases eventually found to be Lynch syndrome do not have family histories that meet these rather rigorous, narrow criteria. More likely in the clinic setting, the clinician is confronted with a single patient whose personal history of early-onset CRC has prompted a more detailed risk assessment. In the complete absence of family history (eg, in case of adoption with closed adoption records), the early onset alone may well be enough to trigger MSI/IHC testing. Otherwise, virtually any additional family history of Lynch syndrome-associated tumors will be enough to prompt MSI/IHC. Occasionally, the history of an earlier personal diagnosis of colorectal or endometrial cancer will raise the question, even if the patient is now older. In other cases, sebaceous tumors may point to the Muir-Torre variant. In still others, the pathology of the resected tumor may raise a suspicion for Lynch syndrome. Perhaps an endoscopic biopsy will have presented an opportunity for MSI/IHC which can be carried out prior to surgical resection and which may support a more aggressive scope of resection (subtotal colectomy as opposed to segmental resection), even if definitive mutational testing cannot be carried out during the window of time between endoscopic diagnosis and surgery.

As the recent data from large series of CRCs from Finland, ${ }^{77}$ the Mayo Clinic, ${ }^{37}$ and Ohio $^{10}$ demonstrate, no one clinical, family history, or pathologic consideration carries with it sufficient sensitivity and specificity for Lynch syndrome as to constitute a sole basis for selection for further testing. For better or worse, informed clinical judgment as to likely yield, coupled with the patient's own threshold for willingness to undergo further evaluation, will determine whether or not MSI/IHC is performed. This approach, which is practiced universally, inevitably leads to severe underdiagnosis of Lynch syndrome. A better approach is to practice some form of molecular screening on all newly diagnosed CRC patients. The feasibility of this approach is being explored. ${ }^{10}$ Further studies, including the use of IHC as the primary screen, and incorporating cost-benefit analyses, will be necessary before general recommendations can be proffered.

If the decision is made to proceed with MSI/IHC and appropriate counseling has been provided, most patients will choose to follow up an informative result with mutational testing. The choice not to pursue mutational testing despite informative MSI/IHC likely reflects poor communications during the initial clinical/genetic counseling experience. The more difficult decision is whether to do further testing if MSI/IHC is not informative. Here, the weight of pretest clinical risk and patient determination make for challenging decision making. When mutational testing is pursued, whether following an informative MSI/ IHC, following an uninformative MSI/IHC, or without the use of MSI/IHC at all (as is the practice of some), even more difficult challenges in interpretation exist. The easy case is a test that is positive for a pathologic MMR gene mutation. Again, if communications have been effective to this point, most subjects will have already decided to inform at-risk relatives of the findings, perhaps aided by educational materials that have been offered by those doing the counseling and/or testing. What to do when the mutational test yields no mutation can be complicated. If, for example, there was loss of MSH2-associated protein, the germline mutation test may well have been a false negative, representing limitations in the testing itself. If additional testing is possible (rearrangement studies, diploid-haploid conversion-aided testing, segregation analysis), then they may be considered. If no prior MSI/ IHC was carried out, one might wish to 'back-track' and do so. If for any reason the mutational testing was considered low yield in the first place (eg, carried out despite uninformative MSI/IHC), one may simply choose to offer empiric surveillance recommendations, that is, to do surveillance in 'at-risk' relatives 'as if' they were carriers. An unfortunately common situation involves mutational testing that yields a variant 'of uncertain significance.'

It is important that colon cancer surveillance be cast in the perspective of general population vs hereditary CRC. Central to these concerns is the fact that hereditary CRC surveillance differs strikingly from general population CRC surveillance guidelines. ${ }^{78}$ As recently as 1990 , there was a marked paucity of guidelines for hereditary CRC surveillance, and no widely endorsed guidelines for general-population CRC surveillance. There was even disagreement among trusted organizations about which tests were recommended or even whether any tests for screening would be appropriate for the general population. Ransohoff ${ }^{78}$ notes that when screening was performed at all, fecal occult blood testing (FOBT) was the most common test, with sigmoidoscopy less frequently used. Colonoscopy was rarely used for surveillance. The question of whether CRC screening of any kind would reduce CRC mortality was still unanswered. 
The situation in 2005 is significantly different. ${ }^{78}$ CRC screening for the general population is known to be effective, and it is now being improved and reimbursed. Colonoscopy has become important as a primary CRC surveillance method. New tests such as virtual colonoscopy are on the horizon. Surveillance for hereditary CRC is also slowly improving. Järvinen et $a l^{65}$ provided convincing evidence that when high-risk individuals who were screened were compared to equally high-risk family members who declined screening, there was a significant increase in early-stage cancer detections and a reduction in deaths.

Finally, Burt and Neklason ${ }^{79}$ conclude that cellular mechanisms which relate to hereditary CRC-causing genes are likely to identify molecular targets for cancer prevention and therapy. Central to this developing approach to hereditary CRC will be the knowledgeable physician who identifies the high-risk individual, educates him/her intensively, and encourages compliance with screening and management recommendations.

\section{Acknowledgements}

This article was supported by revenue from Nebraska cigarette taxes awarded to Creighton University by the Nebraska Department of Health and Human Services. Its contents are solely the responsibility of the authors and do not necessarily represent the official views of the State of Nebraska or the Nebraska Department of Health and Human Services. Support was also given by the Jacqueline Seroussi Memorial Foundation for Cancer Research, by the National Institutes of Health through Grant nos. 1U01 CA 86389-04, P30 CA 16058 and R01 CA 67941. Dr Henry Lynch's work is partially funded through the Charles $F$ and Mary $C$ Heider Chair in Cancer Research, which he holds at Creighton University. Dr de la Chapelle's work is partially funded by the State of Ohio Biomedical and Technology Transfer Commission. The views expressed in this article are those of the authors and do not necessarily reflect the views of the State of Ohio Biomedical and Technology Transfer Commission. Dr Boland is funded by a Grant from the National Cancer Institute, NIH, R01-CA 72851, and an endowed chair from the Baylor Research Institute.

\section{References}

1 International Agency for Research on Cancer: Globocan 2002. Available at: http://www-dep.iarc.fr/ Accessed 06/01/2005.

2 Lynch HT, de la Chapelle A: Genetic susceptibility to nonpolyposis colorectal cancer. J Med Genet 1999; 36: 801-818.

3 Lynch HT, de la Chapelle A: Genomic medicine: hereditary colorectal cancer. $N$ Engl J Med 2003; 348: 919-932.

4 Jass JR: Colorectal adenoma progression and genetic change: is there a link? Ann Med 1995; 27: 301-306.

5 Smyrk TC, Watson P, Kaul K, Lynch HT: Tumor-infiltrating lymphocytes are a marker for microsatellite instability in colorectal cancer. Cancer 2001; 91: 2417-2422.

6 Vogelstein B, Kinzler KW (eds): The genetic basis of human cancer. New York: McGraw-Hill, 1998.

7 Liu B, Parsons RE, Hamilton SR et al: hMSH2 mutations in hereditary nonpolyposis colorectal cancer kindreds. Cancer Res 1994; 54: 4590-4594.

8 Froggatt NJ, Koch J, Davies R et al: Genetic linkage analysis in hereditary non-polyposis colon cancer syndrome. J Med Genet 1995; 32: 352-357.
9 Desai DC, Lockman JC, Chadwick RB et al: Recurrent germline mutation in MSH2 arises frequently de novo. J Med Genet 2000; 37: 646-652.

10 Hampel H, Frankel WL, Martin E et al: Screening for Lynch syndrome (hereditary nonpolyposis colorectal cancer). $N$ Engl J Med 2005; 352: 1851-1860.

11 Nystrom-Lahti M, Kristo P, Nicolaides NC et al: Founding mutations and Alu-mediated recombination in hereditary colon cancer. Nat Med 1995; 1: 1203-1206.

12 Moisio A-L, Sistonen P, Weissenbach J, de la Chapelle A, Peltomaki P: Age and origin of two common MLH1 mutations predisposing to hereditary colon cancer. Am J Hum Genet 1996; 59: $1243-1251$.

13 Foulkes WD, Thiffault I, Gruber SB et al: The founder mutation MSH2*1906 $\mathrm{G} \rightarrow \mathrm{C}$ is an important cause of hereditary nonpolyposis colorectal cancer in the Ashkenazi Jewish population. Am J Hum Genet 2002; 71: 1395-1412.

14 de la Chapelle A: Genetic predisposition to colorectal cancer. Nat Rev Cancer 2004; 4: 769-780.

15 Wagner A, Barrows A, Wijnen JT et al: Molecular analysis of hereditary nonpolyposis colorectal cancer in the United States: high mutation detection rate among clinically selected families and characterization of an American founder genomic deletion of the MSH2 gene. Am J Hum Genet 2003; 72: 1088-1100.

16 Nakagawa $\mathrm{H}$, Hampel $\mathrm{H}$, de la Chapelle A: Identification and characterization of genomic rearrangements of MSH2 and MLH1 in Lynch syndrome (HNPCC) by novel techniques. Hum Mutat 2003; 22: 258.

17 Lynch HT, Coronel SM, Okimoto R et al: A founder mutation of the $\mathrm{MSH} 2$ gene and hereditary nonpolyposis colorectal cancer in the United States. JAMA 2004; 291: 718-724.

18 Wagner A, van der Klift H, Franken P et al: A $10 \mathrm{Mb}$ paracentric inversion of chromosome arm $2 p$ inactivates $\mathrm{MSH} 2$ and is responsible for HNPCC in a North-American kindred. Genes Chromosomes Cancer 2002; 35: 49-57.

19 Lynch HT, de la Chapelle A, Hampel $\mathrm{H}$ et al: The American founder mutation for Lynch syndrome: prevalence estimates and implications. Cancer 2005; 106: 448-452.

20 Gallinger S, Aronson M, Shayan K et al: Gastrointestinal cancers and neurofibromatosis type 1 features in children with a germline homozygous MLH1 mutation. Gastroenterology 2004; 126: 576-585.

21 Laken SJ, Petersen GM, Gruber SB et al: Familial colorectal cancer in Ashkenazim due to a hypermutable tract in APC. Nat Genet 1997; 17: 79-83.

22 Bian Y, Caldes T, Wijnen J et al: TGFBR1*6A may contribute to hereditary colorectal cancer. J Clin Oncol 2005; 23: 3074-3078.

23 Pasche B, Knobloch TJ, Bian Y et al: Somatic acquisition and signaling of TGFBR1*6A in cancer. JAMA 2005; 294: 1634-1646.

24 Jones JS, Chi X, Gu X et al: p53 polymorphism and age of onset of hereditary nonpolyposis colorectal cancer in a Caucasian population. Clin Cancer Res 2004; 10: 5845-5849.

25 Kruger S, Bier A, Engel C et al: The p53 codon 72 variation is associated with the age of onset of hereditary non-polyposis colorectal cancer (HNPCC). J Med Genet 2005; 42: 769-773.

26 Sotamaa K, Liyanarachchi S, Mecklin JP et al: p53 codon 72 and MDM2 SNP309 polymorphisms and age of colorectal cancer onset in Lynch syndrome. Clin Cancer Res 2005; 11: 6840-6844.

27 Vasen HFA, Boland CR: Progress in genetic testing, classification, and identification of Lynch syndrome. JAMA 2005; 293: $2028-2930$.

28 Rodriguez-Bigas MA, Boland CR, Hamilton SR et al: A National Cancer Institute workshop on hereditary nonpolyposis colorectal cancer syndrome: meeting highlights and Bethesda Guidelines. J Natl Cancer Inst 1997; 89: 1758-1762.

29 Ionov YM, Peinado MA, Malkhosyan S, Shibata D, Perucho M: Ubiquitous somatic mutations in simple repeated sequences reveal a new mechanism for colonic carcinogenesis. Nature 1993; 363: $558-561$. 
30 Peltomäki P, Aaltonen L, Sistonen P et al: Genetic mapping of a locus predisposing to human colorectal cancer. Science 1993; 260: $810-812$.

31 Aaltonen LA, Peltomaki P, Leach FS et al: Clues to the pathogenesis of familial colorectal cancer. Science 1993; 260: 812-816.

32 Thibodeau SN, Bren G, Schaid D: Microsatellite instability in cancer of the proximal colon. Science 1993; 260: 816-819.

33 Lin KM, Shashidharan M, Thorson AG et al: Cumulative incidence of colorectal and extracolonic cancers in MLH1 and MSH2 mutation carriers of hereditary nonpolyposis colorectal cancer. J Gastrointest Surg 1998; 2: 67-71.

34 Lynch HT, Lynch PM: Molecular screening for the Lynch syndrome - better than family history? $N$ Engl J Med 2005; 352: $1920-1922$.

35 Hendriks Y, Franken P, Dierssen JW et al: Conventional and tissue microarray immunohistochemical expression analysis of mismatch repair in hereditary colorectal tumors. Am J Pathol 2003; 162: $469-477$.

36 Guttmacher AE, Collins FS, Carmona RH: The family history more important than ever. N Eng J Med 2004; 351: 2333-2336.

37 Lindor NM, Burgart LJ, Leontovich $\mathrm{O}$ et al: Immunohistochemistry versus microsatellite instability testing in phenotyping colorectal tumors. J Clin Oncol 2002; 20: 1043-1048.

38 Piñol V, Castells A, Andreu M et al: Accuracy of revised Bethesda guidelines, microsatellite instability, and immunohistochemistry for the identification of patients with hereditary nonpolyposis colorectal cancer. IAMA 2005; 293: 1986-1994.

39 Watson P, Ashwathnarayan R, Lynch HT, Roy HK: Tobacco use and increased colorectal cancer risk in patients with hereditary nonpolyposis colorectal cancer (Lynch syndrome). Arch Intern Med 2004; 164: 2429-2431.

40 Arnold CN, Goel A, Boland CR: Role of hMLH1 promoter hypermethylation in drug resistance to 5-fluorouracil in colorectal cancer cell lines. Int J Cancer 2003; 106: 66-73.

41 Aebi S, Kurdi-Haider B, Gordon R et al: Loss of DNA mismatch repair in acquired resistance to cisplatin. Cancer Res 1996; 56: 3087-3090.

42 Magrini R, Bhonde MR, Hanski ML et al: Cellular effects of CPT-11 on colon carcinoma cells: dependence on p53 and hMLH1 status. Int J Cancer 2002; 101: 23-31.

43 Gryfe R, Kim H, Hsieh ETK et al: Tumor microsatellite instability and clinical outcome in young patients with colorectal cancer. $N$ Engl J Med 2000; 342: 69-77.

44 Ribic CM, Sargent DJ, Moore MJ et al: Tumor microsatelliteinstability status as a predictor of benefit from fluorouracil-based adjuvant chemotherapy for colon cancer. N Engl J Med 2003; 349: 247-257.

45 Carethers JM, Smith EJ, Behling CA et al: Use of 5-fluorouracil and survival in patients with microsatellite-unstable colorectal cancer. Gastroenterology 2004; 126: 394-401.

46 de Vos tot Nederveen Cappel WH, Meulenbeld HJ, Kleibeuker JH et al: Survival after adjuvant 5-FU treatment for Stage III colon cancer in hereditary nonpolyposis colorectal cancer. Int J Cancer 2004; 109: 468-471.

47 Johnson L, Chu E: Lack of benefit of 5-fluorouracil-based adjuvant chemotherapy in colorectal cancer with microsatellite instability. Clin Colorectal Cancer 2002; 2: 146-148.

48 Umar A, Boland CR, Terdiman JP et al: Revised Bethesda Guidelines for hereditary nonpolyposis colorectal cancer (Lynch syndrome) and microsatellite instability. J Natl Cancer Inst 2004; 96: $261-268$.

49 Fallik D, Borrini F, Boige V et al: Microsatellite instability is a predictive factor of the tumor response to irinotecan in patients with advanced colorectal cancer. Cancer Res 2003; 63: $5738-5744$.

50 Lynch HT: Family information service and hereditary cancer. Cancer 2001; 91: 625-628.

51 American Society of Human Genetics: ASHG statement: professional disclosure of familial genetic information. Am J Hum Genet 1998; 62: 474-483.
52 Hampel H, Stephens JA, Pukkala E et al: Cancer risk in hereditary nonpolyposis colorectal cancer syndrome: later age of onset. Gastroenterology 2005; 129: 415-421.

53 Lindor NM, Rabe K, Petersen GM et al: Lower cancer incidence in Amsterdam-I criteria families without mismatch repair deficiency: familial colorectal cancer type X. JAMA 2005; 293: $1979-1985$

54 Lynch HT, Kimberling W, Albano WA et al: Hereditary nonpolyposis colorectal cancer (Lynch syndromes I and II). I. Clinical description of resource. Cancer 1985; 56: 934-938.

55 Lynch HT, Schuelke GS, Kimberling WJ et al: Hereditary nonpolyposis colorectal cancer (Lynch syndromes I and II). II. Biomarker studies. Cancer 1985; 56: 939-951.

56 Broaddus RR, Lynch PM, Lu KH, Luthra R, Michelson SJ: Unusual tumors associated with the hereditary nonpolyposis colorectal cancer syndrome. Mod Pathol 2004; 17: 981-989.

57 Sijmons R, Hofstra R, Hollema $\mathrm{H}$ et al: Inclusion of malignant fibrous histiocytoma in the tumour spectrum associated with hereditary non-polyposis colorectal cancer. Genes Chromosomes Cancer 2000; 29: 353-355.

58 den Bakker MA, Seynaeve C, Kliffen M, Dinjens WN: Microsatellite instability in a pleomorphic rhabdomyosarcoma in a patient with hereditary nonpolyposis colorectal cancer. Histopathology 2003; 43: 297-299.

59 Suwa K, Ohmori M, Miki H: Microsatellite alterations in various sarcomas in Japanese patients. J Orthop Sci 1999; 4: 223-230.

60 Kawaguchi K, Oda T, Takahira T et al: Microsatellite instability and hMLH1 and hMSH2 expression analysis in soft tissue sarcomas. Oncol Rep 2005; 13: 241-246.

61 Soravia C, van der Klift H, Brundler MA et al: Prostate cancer is part of the hereditary non-polyposis colorectal cancer (HNPCC) tumor spectrum. Am J Med Genet 2003; 121A: 159-162.

62 Lynch PM, Lynch HT, Harris RE: Hereditary proximal colonic cancer. Dis Colon Rectum 1977; 20: 661-668.

63 Rijcken FEM, Hollema H, Kleibeuker JH: Proximal adenomas in hereditary non-polyposis colorectal cancer are prone to rapid malignant transformation. Gut 2002; 50: 382-386.

64 Lynch HT, Casey MJ, Lynch J, White TEK, Godwin AK: Genetics and ovarian carcinoma. Semin Oncol 1998; 25: 265-281.

65 Järvinen HJ, Aarnio M, Mustonen $\mathrm{H}$ et al: Controlled 15-year trial on screening for colorectal cancer in families with hereditary nonpolyposis colorectal cancer. Gastroenterology 2000; 118: 829-834.

66 Vasen HFA, Nagengast FM, Khan PM: Interval cancers in hereditary non-polyposis colorectal cancer (Lynch syndrome). Lancet 1995; 345: 1183-1184.

67 Church JM: Prophylactic colectomy in patients with hereditary nonpolyposis colorectal cancer. Ann Med 1996; 28: 479-482.

68 Lynch HT: Is there a role for prophylactic subtotal colectomy among hereditary nonpolyposis colorectal cancer germline mutation carriers? Dis Colon Rectum 1996; 39: 109-110.

69 Parsons R, Li GM, Longley MJ et al: Hypermutability and mismatch repair deficiency in RER+ tumor cells. Cell 1993; 75: $1227-1236$

70 Koi M, Umar A, Chauhan DP et al: Human chromosome 3 corrects mismatch repair deficiency and microsatellite instability and reduces $\mathrm{N}$-methyl- $\mathrm{N}$-nitro- $\mathrm{N}$-nitrosoguanidine tolerance in colon tumor cells with homozygous hMLH1 mutation (published erratum appears in Cancer Res 55:201, 1995). Cancer Res 1994; 54: $4308-4312$

71 Hawn MT, Umar A, Carethers JM et al: Evidence for a connection between the mismatch repair system and the $\mathrm{G}_{2}$ cell cycle checkpoint. Cancer Res 1995; 55: 3721-3725.

72 Carethers JM, Hawn MT, Chauhan DP et al: Competency in mismatch repair prohibits clonal expansion of cancer cells treated with $N$-methyl- $N^{\prime}$-nitro- $N$-nitrosoguanidine. J Clin Invest 1996; 98: 199-206.

73 Branch P, Aquilina G, Bignami M, Karran P: Defective mismatch binding and a mutator phenotype in cells tolerant to DNA damage. Nature 1993; 362: 652-654 
74 Branch P, Aquilina G, Hess P, Bignami M, Karran P: Mammalian cells defective in DNA mismatch correction. Ann NY Acad Sci 1994; 726: 355-358.

75 Branch P, Hampson R, Karran P: DNA mismatch binding defects, DNA damage tolerance, and mutator phenotypes in human colorectal carcinoma cell lines. Cancer Res 1995; 55: 2304-2309.

76 Elsaleh H, Joseph D, Grieu F, Spry N, Iacopetta B: Association of tumour site and sex with survival benefit from adjuvant chemotherapy in colorectal cancer. Lancet 2000; 355: 1745-1750.

77 Aaltonen LA, Salovaara R, Kristo P et al: Incidence of hereditary nonpolyposis colorectal cancer and the feasibility of molecular screening for the disease. $N$ Engl J Med 1998; 338: 1481-1487.

78 Ransohoff DF: Colon cancer screening in 2005: status and challenges. Gastroenterology 2005; 128: 1685-1695.
79 Burt R, Neklason DW: Genetic testing for inherited colon cancer. Gastroenterology 2005; 128: 1696-1716.

80 Vasen HFA, Mecklin J-P, Meera Khan P, Lynch HT: The International Collaborative Group on Hereditary Nonpolyposis Colorectal Cancer (ICG-HNPCC). Dis Colon Rectum 1991; 34: 424-425.

81 Vasen HFA, Watson P, Mecklin J-P, Lynch HT, ICG-HNPCC: New clinical criteria for hereditary nonpolyposis colorectal cancer (HNPCC, Lynch syndrome) proposed by the International Collaborative Group on HNPCC. Gastroenterology 1999; 116: $1453-1456$.

82 Laghi L, Bianchi P, Roncalli M, Malesci A: Revised Bethesda guidelines for hereditary nonpolyposis colorectal cancer (Lynch syndrome) and microsatellite instability. J Natl Cancer Inst 2004; 96: $1402-1403$. 